\title{
New SERS-active junction based on cerium dioxide facet dielectric films for biosensing
}

\author{
Ilya Kurochkin ${ }^{1 *}$, Ilya Ryzhikov², Andrey Sarychev², Konstantin Afanasiev², Igor Budashov', \\ Marina Sedova ${ }^{2}$, Irina Boginskaya ${ }^{2}$, Sergey Amitonov ${ }^{4}$ and Andrey Lagarkov ${ }^{2}$
}

\author{
${ }^{1}$ Faculty of Chemistry, Lomonosov Moscow State University, Moscow, Russia \\ ${ }^{2}$ Institute for Theoretical and Applied Electromagnetics, The Russian Academy of Science, Moscow, Russia \\ ${ }^{3}$ Emanuel Institute of Biochemical Physics, The Russian Academy of Science, Moscow, Russia \\ ${ }^{4}$ Faculty of Physics, Lomonosov Moscow State University, Moscow, Russia \\ *corresponding author, E-mail: i kur@genebee.msu.su
}

\begin{abstract}
Further enhance of the Raman scattering is the priority for the development of the modern molecular diagnostic methods. Expected increasing in detection sensitivity of the biological and chemical agents provides substantial progress in such areas as: proteomics (discovery of new disease markers), pharmacokinetics of drugs, analysis of toxins and infections agents, drug analysis, food safety, and environmental safety.

In this paper we investigated the possibility of the facet structures, based on cerium dioxide to further enhance the SERS signal. During the studies a new metamaterial was developed. The metamaterial is based on the facet cerium dioxide films and plasmonic nanoparticles that are immobilized on its surface. The new metamaterial provides additional SERS signal amplification factor of 211. Thus developed material offers the prospect of increasing the sensitivity and selectivity of biochemical and immunological analysis.
\end{abstract}

\section{Introduction}

Over the last decade, many research efforts have been focused on the development the fast, sensitive, and low cost sensors, especially for biological and chemical agents. Emerging advances in plasmonics, nanotechnology, surface engineering, and optics open an opportunity of using the near-field interactions to significantly increase the optical signal strength.

It is known that the media with random distribution of the refractive index and non-uniform rough surface provide the inhomogeneous distribution of the intensity maxima in transmitted and reflected light [1]. Recently, we have shown that resonant interactions in the special dielectric metamaterials (facet dielectric films) yield significantly enhanced local fields covering large surface areas [2]. In this report we consider the possibility of combining plasmon resonances in metal (gold) nanoparticles with localized electromagnetic resonaces in the facet dielectric films of cerium dioxide for highly sensitive SERS detection of chemical compounds and biological agents.

\section{Results}

\subsection{Preparation of facet cerium dioxide films}

A technological route to facet cerium dioxide films consists of the following:

- pretreatment of surface of a polycor substrate by washing in isopropyl alcohol and ion cleaning in vacuum chamber $\left(10^{-2}\right.$ Torr) by an ion beam (beam current $150 \mathrm{~mA}$, voltage $1.5 \mathrm{kV}$ ) for 15 minutes;

- deposition by electron beam evaporation of aluminum sublayer, thickness of $100-150 \mathrm{~nm}$, current in the electron beam was $100 \mathrm{~mA}$, voltage $-8 \mathrm{kV}$;

- cerium dioxide of high purity was placed in a water-cooled copper crucible, electron-beam evaporation was carried out at a beam current of $30 \mathrm{~mA}$ and a voltage of $8 \mathrm{kV}$.

The thickness of the deposited films was controlled by optical inspection of the interference maxima and minima of the transmittance at a wavelength of $900 \mathrm{~nm}$ on the control glass samples.

\subsection{Preparation and immobilization of Au- nanoparticles on the facet cerium dioxide films}

Au-nanoparticles (Au-NP) were prepared by well-known citrate method [3]. Au-Np size was determined by Nanoparticle Tracking Analysis (average size - 56+1 nm). For the preparation of SERS active particles, Au-NPs were modified by 3,3'-dithio-bis(6-nitrobenzoic acid) - (DTNB) in accordance with the scheme shown in Fig. 1.

$\mathrm{Au}-\mathrm{NPs}$ were adsorbed onto facet cerium dioxide films after deposition of polycation (poly(diallyldimethylammonium chloride)) according to the procedure described in the article [4].

\subsection{Morphology and structure of cerium dioxide films}

Morphology of cerium dioxide films were studied by scanning electron microscopy (SEM) using a Quanta microscope (FEI) with resolution near $5 \mathrm{~nm}$. Representative 
SEM image and cross section of cerium dioxide film are shown in Fig. 2.

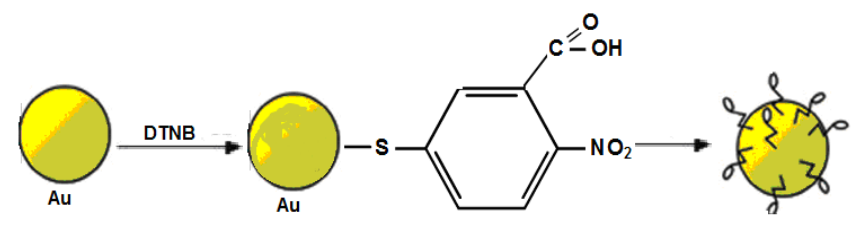

Figure 1: Scheme for preparation of Au-NP - DTNB conjugates.

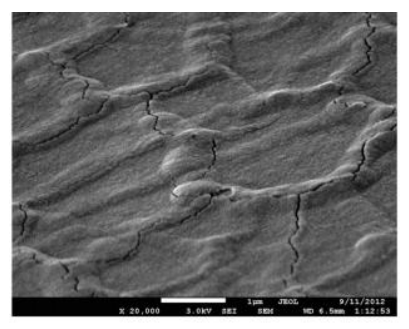

a

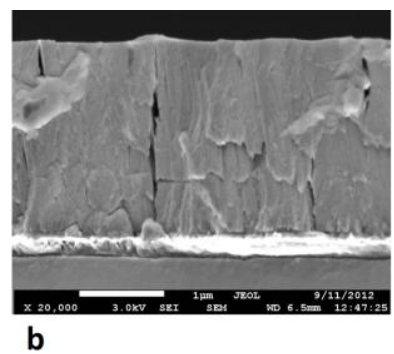

Figure 2: (a) General SEM view of the $\mathrm{CeO} 2$ structure; (b)cross section of $\mathrm{CeO} 2$ film.

Presented in Fig. 2 images show characteristic faceted structure of the films. A detailed study of the structure facet films was conducted by atomic force microscopy. Dedicated as a result the basic elements of this structure are reflected in the scheme shown in Fig. 3.

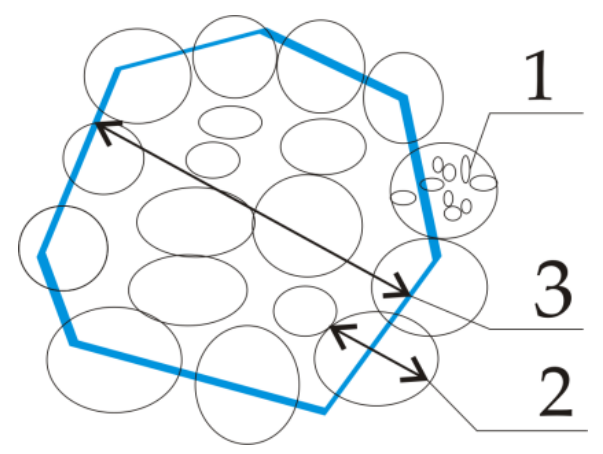

Figure 3: The general scheme of the resulting facet structure.
Nanoscale structures (1) form larger agglomerates (2) with characteristic dimensions of several hundred nanometers. Agglomerates are arranged so that they form a facet (3). Perimeter facet (blue line) forms a kind of curb. The facets are separated from each other by small cracks (several nanometers). Estimated by AFM dimensions for selected structures are shown in Table 1.

Table 1: Dimensions of the elements of the facet structures at various cerium dioxide film thicknesses.

\begin{tabular}{ccccc}
$\begin{array}{c}\text { Film } \\
\text { thickness, } \\
\mathrm{nm}\end{array}$ & $\begin{array}{c}\text { Curb } \\
\text { height, } \\
\mathrm{nm}\end{array}$ & $\begin{array}{c}\text { Facet } \\
\text { size, } \\
\mu \mathrm{m}\end{array}$ & $\begin{array}{c}\text { Agglo- } \\
\text { merate } \\
\text { size, } \mu \mathrm{m}\end{array}$ & $\begin{array}{c}\text { Nano } \\
\text { struct. } \\
\text { size, } \\
\mathrm{nm}\end{array}$ \\
\hline 800 & $29 \pm 4$ & $1,3 \pm 0,1$ & $0,32 \pm 0,03$ & $28 \pm 2$ \\
1200 & $50 \pm 7$ & $1,5 \pm 0,1$ & $0,37 \pm 0,04$ & $29 \pm 4$ \\
1600 & $118 \pm 17$ & $2,0 \pm 0,1$ & $0,45 \pm 0,04$ & $32 \pm 4$ \\
2000 & $126 \pm 15$ & $2,5 \pm 0,3$ & $0,57 \pm 0,04$ & $23 \pm 3$ \\
2400 & $105 \pm 24$ & $2,3 \pm 0,3$ & $0,60 \pm 0,05$ & $25 \pm 4$ \\
2800 & $128 \pm 19$ & $2,8 \pm 0,2$ & $0,65 \pm 0,06$ & $75 \pm 7$ \\
\hline
\end{tabular}

The Table 1 shows that nanoscale structures size remains constant for all thickness except $2800 \mathrm{~nm}$ where average size is higher in 3 times. Increase in film thickness leads to an increase in the size of the agglomerates curb height and facet size. A slight deviation from this rule is observed at $2400 \mathrm{~nm}$ film thickness.

Investigation of the optical images and the distribution of Raman signal on the cerium dioxide films was performed using confocal Raman imaging system Alpha 500R (WITec, Germany). Raman scattering system registration was equipped with WITec inverted confocal microscope. It is situated at solid granite plate mounted at active vibration isolation system. Special XY positioner provide accuracy moving of sample table. Microscope objective is EC Epiplan-Neofluar 100x/0,9 DIC $\infty / 0$ Carl Zeizz objective. Objective numerical aperture consists 0,9 . Light source is green laser WITec with $532 \mathrm{~nm}$ wave length and its maximal power is $60 \mathrm{~mW}$. Measurement was carried out at 5 $\mathrm{mW}$ of all laser power. Raman scattering light from sample enters the optical fiber and goes to the spectrometer. Signal registration is performed by Andor CCD camera. WITec Raman scattering system provides $90 \%$ delivery of light to the detector.

Fig. 4 show optical image and Raman intensity distribution at $456 \mathrm{~cm}^{-1}$ (characteristic Raman shift for cerium dioxide). 


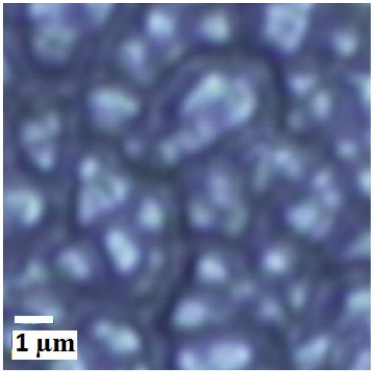

a

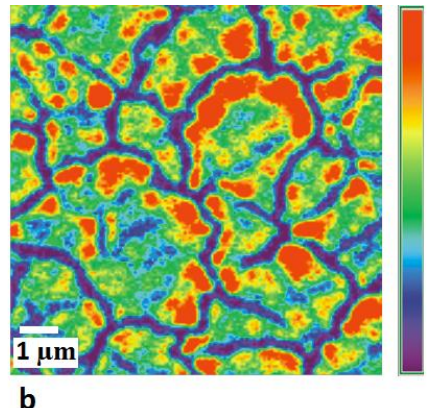

Figure 4: (a) Optical image of $2400 \mathrm{~nm} \mathrm{CeO} 2$ surface; (b) intensity distribution maps for mainRaman peak of 2400 nm CeO2. Red - 300 CCD counts, violet - 1700 CCD counts.

These images show the irregular distribution of the signal on the film surface. Areas of more intense signal located mainly in the border area of facets.

\subsection{Morphology of SERS-active junction of Au-NP and facet cerium dioxide films}

Morphology of cerium dioxide films after Au-NP deposition were studied by SEM using a Supra-40 microscope (Carl Zeiss). Representative SEM images are shown in Fig. 5.

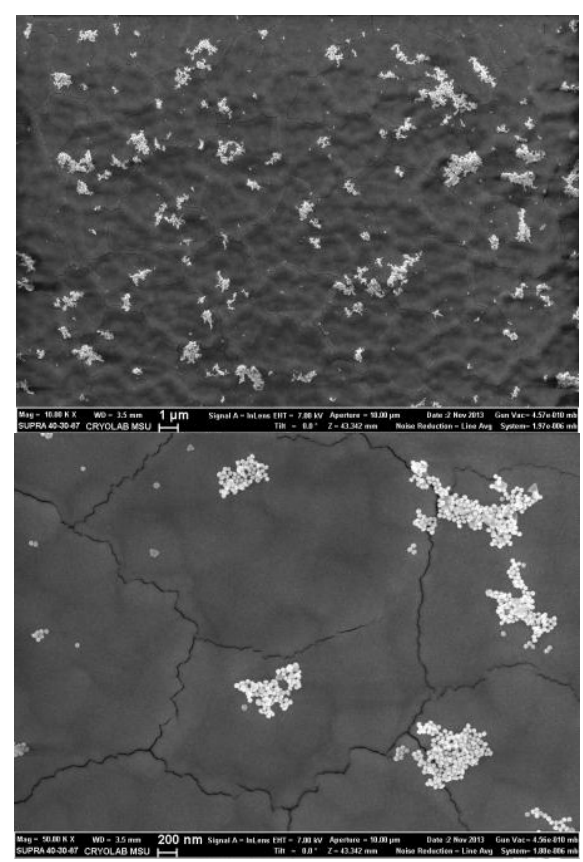

Figure 5: SEM images of facet cerium dioxide films with Au-NP.

The fraction of the surface occupied by the gold nanoparticles after deposition to the cerium dioxide surface was evaluated using software Gwyddion. Data for films with different thickness are given in Table 2.

Table 2: Fraction of surface occupied by the gold nanoparticles after deposition to the cerium dioxide films.

\begin{tabular}{cc}
\hline $\begin{array}{c}\text { Film } \\
\text { thickness, } \\
\text { nm }\end{array}$ & $\begin{array}{c}\text { Fraction of } \\
\text { surface for } \\
\text { gold NP [\%] }\end{array}$ \\
\hline 90 & $5.7 \pm 0.7$ \\
200 & $5.8 \pm 0.7$ \\
300 & $4.6 \pm 0.7$ \\
400 & $3.8 \pm 0.7$ \\
500 & $1.3 \pm 0.7$ \\
600 & $3.7 \pm 0.8$ \\
700 & $2.3 \pm 1.1$ \\
800 & $4.5 \pm 1.2$ \\
1200 & $7.7 \pm 4.6$ \\
1600 & $6.0 \pm 3.7$ \\
2000 & $5.2 \pm 0.6$ \\
2400 & $2.3 \pm 1.1$ \\
2800 & $2.1 \pm 2.7$
\end{tabular}

From Table 2 it follows that immobilization of Au-NPs lead to covering from 1 to 8 percent of the cerium dioxide surface.

\subsection{SERS spectra}

A Raman spectrometer innoRam, model BWS445(B)-785S (B\&W Tek, Inc.) with continuous-wave laser-785 (290 $\mathrm{mW}$ ) was used for the collection of spectra using an excitation wavelength of $785 \mathrm{~nm}$. The excitation was performed in an epi configuration through a $20 \mathrm{X}$ objective $(\mathrm{NA}=0.4)$ on a microscope. The SERS signal was collected through the same objective in 180 degree backscattering geometry. Laser power for all experiments was $29 \mathrm{~mW}$. The acquisition time was 1.5 seconds. $326 \mathrm{~cm}^{-}$ $1,1060 \mathrm{~cm}^{-1}, 1338 \mathrm{~cm}^{-1}$ and $1558 \mathrm{~cm}^{-1}$ Raman scattering bands of thio 6-nitrobenzoic acid [5] were used to assess the effectiveness of the SERS signal. SERS signal intensity of these bands was calculated as the average of the measurements in the ten points of the sample after subtracting the baseline signal.

\section{Discussion}

Studies have shown that in the process of formation of cerium dioxide films as a result apparently of relaxation of tensions arising formed facet structure with a characteristic size about 2-3 microns. Irregular distribution of the Raman signal on the film surface (see Fig. 4 ) indicates the possibility of an additional signal amplification after immobilization SERS active structures on the surface of the facet structures due to the presence of local amplification of the electromagnetic field.

Study of SERS signal intensity from DTNB - Au-NP on cerium dioxide films with various thicknesses showed that the magnitude of the signal oscillates with increasing film thickness (see Fig. 6). 


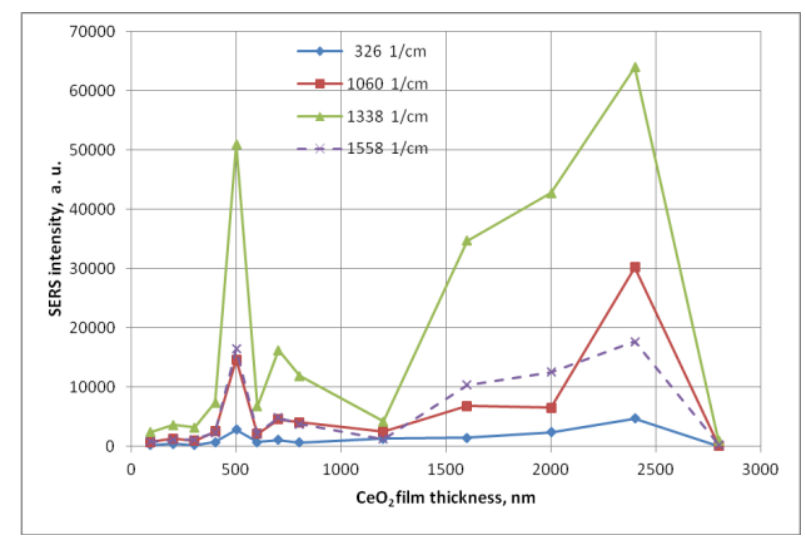

Figure 6: SERS signal at different Raman scattering bands from DTNB - Au-NP on cerium dioxide films with various thicknesses after normalization to the amount of gold nanoparticles.

Normalization of SERS signals on the magnitude of the signal received at a film thickness of $2800 \mathrm{~nm}$ (minimal signal), makes it possible to estimate the values of the coefficients of additional enhancing for the SERS signal. The data after the appropriate transformation shown in Fig. 7.

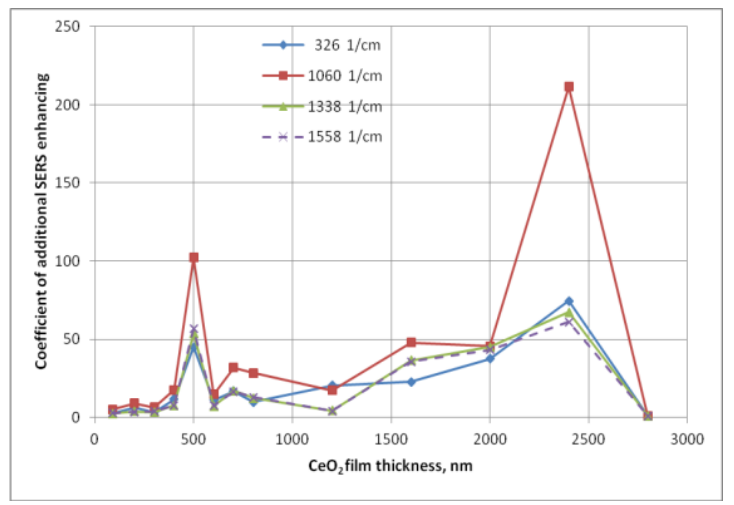

Figure 7: SERS signal at different Raman scattering bands from DTNB - Au-NP on cerium dioxide films with various thicknesses after normalization to the signal from films with a thickness of $2800 \mathrm{~nm}$.

Maximum, more than 200 times, additional enhancing for the SERS signal is achieved at a film thickness of $2400 \mathrm{~nm}$ and Raman shift $1060 \mathrm{~cm}^{-1}$. It should be noted that the signal enhancement depends on the selected SERS shift as well. In other words, the films of different thickness have a different selectivity relative to the vibrations of different frequencies.

\section{Conclusions}

A new metamaterial based on facet cerium dioxide films and immobilized on its surface plasmonic nanoparticles have been developed. The new material provides additional SERS signal amplification factor of 211 . Using the developed material offers the prospect of increasing the sensitivity and selectivity for enzymatic and immunological assays using thiol-containing substrates and conjugates of antibodies with gold nanoparticles .

\section{Acknowledgements}

This research was supported by Lomonosov Moscow State University Programm of Development and Programm №3 of BEMMBCS of RAS and RFFI grant 12-02-01365-a.

\section{References}

[1] Ed. By J.C. Dainty, Laser Specle and Related Phenomena, Springer-Verlag, Berlin, Heidelberg, New York, 1975.

[2] I.N. Kurochkin, A.K. Sarychev, I.A. Ruzhikov, I.A. Budashov, S.S. Maklakov, S.O. Boyarintsev, A.N. Lagarkov, Surface-enhanced raman scattering-based biosensors // Portable Biosensing of Food Toxicants and Environmental Pollutants. - Series in Sensors. 6000 Broken Sound Parkway NW, STE 300, BOCA RATON, USA, FL, 33487-2742: 2013. - P. 97-123.

[3] J. Turkevich, P.S. Stevenson, J. Hiller, A study of the nucleation and growth processes in the synthesis of colloidal gold, Discuss. Faraday Soc. 11: 55-75, 1955.

[4] M.S. Gromova, L.V. Sigolaeva, M.A. Fastovets, E.G. Evtushenko, I.A. Babin, D.V. Pergushov, S.V. Amitonov, A.V. Eremenko, I.N. Kurochkin, Improved adsorption of choline oxidase on a polyelectrolyte LBL film in the presence of iodine anions, Soft Matter 7: 7404-7409, 2011.

[5] U. Tamer, I.H. Boyac1, E. Temur, A. Zengin, I. Dincer, Y. Elerman, Fabrication of magnetic gold nanorod particles for immunomagnetic separation and SERS application, J. Nanopart. Res., 13:3167-3176, 2011. 